\title{
Perconditioning combined with postconditioning on kidney ischemia and reperfusion ${ }^{1}$
}

Felipe Lobato da Silva Costa', Vitor Nagai Yamaki", Renan Kleber Costa Teixeira'", Daniel Haber Feijólv, André Lopes Valentev , Luan Teles Ferreira de Carvalhov, Edson Yuzur Yasojimavi, Marcus Vinicius Henriques BritovII

'MD, Department of Experimental Surgery, School of Medicine, Universidade do Estado do Pará (UEPA), Belem-PA, Brazil. Conception, design, intellectual and scientific content of the study; interpretation of data; manuscript writing.

"MD, School of Medicine, UEPA, Belem-PA, Brazil. Acquisition and interpretation of data, statistical analysis.

'I'Fellow Master degree, Department of Experimental Surgery, UEPA, Belem-PA, Brazil. Interpretation of data, manuscript writing, critical revision.

IVGraduate student, School of Medicine, UEPA, Belem-PA, Brazil. Interpretation of data, manuscript preparation.

${ }^{\vee}$ Graduate student, School of Medicine, UEPA, Belem-PA, Brazil. Acquisition and interpretation of data, manuscript preparation.

${ }^{V I P h D}$, Associate Professor, Department of Experimental Surgery, School of Medicine, UEPA, Belem-PA, Brazil. Conception, design, intellectual and scientific content of the study; critical revision.

VIIPhD, Full Professor, Department of Experimental Surgery, School of Medicine, UEPA, Belem-PA, Brazil. Conception, design, intellectual and scientific content of the study; critical revision.

\section{Abstract}

Purpose: To evaluate if combination of perconditioning and postconditioning provides improved renal protection compared to perconditioning alone in a model of renal reperfusion injury.

Methods: Thirty rats were assigned into 6 groups: normality; sham; ischemia and reperfusion; postconditioning; perconditioning; perconditioning + postconditioning. Animals were subjected to right nephrectomy and left renal ischemia for 30 minutes. Postconditioning consisted of 3 cycles of 5 min renal perfusion followed by 5 min of renal ischemia after major ischemic period. Perconditioning consisted of 3 cycles of $5 \mathrm{~min}$ hindlimb ischemia followed by 5 min of hindlimb perfusion contemporaneously to renal major ischemic period. After 24 hours, kidney was harvested and blood collected to measure urea and creatinine.

Results: Perconditioning obtained better values for creatinine and urea level than only postconditioning $(p<0.01)$; performing both techniques contemporaneously had no increased results ( $p>0.05)$. Regarding tissue structure, perconditioning was the only technique to protect the glomerulus and tubules $(p<0.05)$, while postconditioning protected only the glomerulus $(p<0.05)$. Combination of both techniques shows no effect on glomerulus or tubules $(p>0.05)$. Conclusions: Perconditioning had promising results on ischemia and reperfusion induced kidney injury, enhanced kidney function and protected glomerulus and tubules. There was no additive protection when postconditioning and perconditioning were combined.

Key words: Ischemic Postconditioning. Ischemia. Reperfusion. Rats. 


\section{Introduction}

After tissue ischemia is resolved and blood flow is restored, the reperfusion injury has been identified as an important mechanism contributing to tissue injury; being even more deleterious than ischemia ${ }^{1,2}$. The clinical syndrome of ischemia and reperfusion (IR) is associated with deleterious consequences for several organs. This syndrome contributes to morbidity and mortality in up to $60-70 \%$ of all cases of acute kidney injury syndrome which constitutes a serious clinical problem that, despite being common, lacks a truly effective treatment $^{3,4}$.

The most critical factor that determines the severity of tissue damage caused by IR appears to be the duration of ischemia ${ }^{5,6}$. In addition to early reperfusion, "tissue conditioning" by a series of alternating intervals of brief episodes of ischemia and reperfusion is currently the most promising approach to limit tissue damage caused by prolonged ischemia ${ }^{7-9}$.

In a setting of kidney transplantation or contrast induced nephropathy, where the renal intervention or damage is planned, the ischemic preconditioning, that consist of performing short cycles of ischemia and reperfusion before a major period of ischemia, could easily be applied and showed great results and applicability ${ }^{10,11}$. However, in kidney transplantation when the organ is from a deceased donor and in a setting of renal artery thrombosis, the preconditioning is not feasible. In such situations, the local ischemic postconditioning (POS) stands pronounced importance. It consists of short cycles of reperfusion and ischemia before the free reperfusion of a tissue that has been under ischemia ${ }^{12}$. POS has been demonstrated to be similarly effective as preconditioning ${ }^{13,14}$.

Schmidt et al. ${ }^{15}$ demonstrated the concept of remote ischemic perconditioning (PER). They applied a tourniquet to a porcine limb to produce alternating periods of occlusion and reperfusion while the myocardium was under ischemia. This technique led to a pronounced protection to the myocardium against the deleterious effects of the ischemia and reperfusion. Since then, this technique has been demonstrated to protect brain, liver, myocardium and kidney from the IR syndrome in various animal models and in clinical settings ${ }^{16-21}$.

PER might be even more practical than POS, because it is performed in a distant tissue and is a non-invasive procedure, thus does not require direct access to the artery and could be easily applied during endovascular revascularization ${ }^{18,22}$. Mechanisms underlying the remote ischemic conditioning are barely understood. It is known that its protective effects are mediated through potassium channels $^{15}$, and through the cellular activation of reperfusion injury salvage kinase pathway and of the survivor activating factor enhancement ${ }^{23}$. The connection between the tissue where the intermittent ischemia is performed and the tissue that is under permanent ischemia appears to occur through a neurogenic pathway, mediated by the parasympathetic system ${ }^{24}$.

PER and POS appear to share similar pathways, such as the activation of the reperfusion injury salvage kinase pathway ${ }^{21,23}$. However, previous studies demonstrated that they might also have unique mechanisms. Moreover, their combination might increase the outcome protection against ischemia and reperfusion injury. Thus, we tested the hypothesis that the combination of PER and POS provides improved renal protection compared to PER alone in a well-established rat model of renal reperfusion injury.

\section{Methods}

The project was previously approved by 
the Animal Use and Care Committee, UEPA.

Thirty (8-10 weeks) male Wistar rats (Rattus norvergicus), weighing 250$300 \mathrm{~g}$, were used in this study. The animals were kept in a vivarium of the Experimental Surgery Laboratory, UEPA with a controlled environment; water and the food were provided ad libitum. The research followed the rules of Brazilian Law for Animal Care (Law: $11.794 / 08$ ) that is based on NIH guidelines, and followed the rules of Council for International Organization of Medical Sciences ethical code for animal experimentation.

The experimental protocol is illustrated in Figure 1. The animals were randomly assigned into the following 6 groups ( $N=5$ for each group):

(1) In the Normality group (N) no intervention was performed. This group served to establish physiologic values for the outcome parameters for the rat strain/colony used in our study;

(2) In the sham Group (SHAM) the same surgical procedure as in the remaining groups was performed but no renal ischemia was induced;

(3) In the ischemia and reperfusion group (IR) renal ischemia was induced for 30 min followed by reperfusion without any form of conditioning;

(4) In the local ischemic postconditioning group (POS) 30 min of renal ischemia was followed by $30 \mathrm{~min}$ of autologous postconditioning ( 3 cycles of $5 \mathrm{~min}$ renal perfusion followed by 5 min of renal ischemia);

(5) In the remote ischemic perconditioning group (PER) renal ischemia was simultaneously accompanied by remote ischemic conditioning. Remote ischemic conditioning consisted of 3 cycles of $5 \mathrm{~min}$ hindlimb ischemia followed by $5 \mathrm{~min}$ of hindlimb perfusion ${ }^{22}$;

(6) In the ischemic remote ischemic perconditioning group + local postconditioning group (PER+POS) renal ischemia was simultaneously accompanied by remote ischemic conditioning in the left hindlimb followed by autologous ischemic postconditioning ( 3 cycles of 5 min renal perfusion followed by $5 \mathrm{~min}$ of renal ischemia).

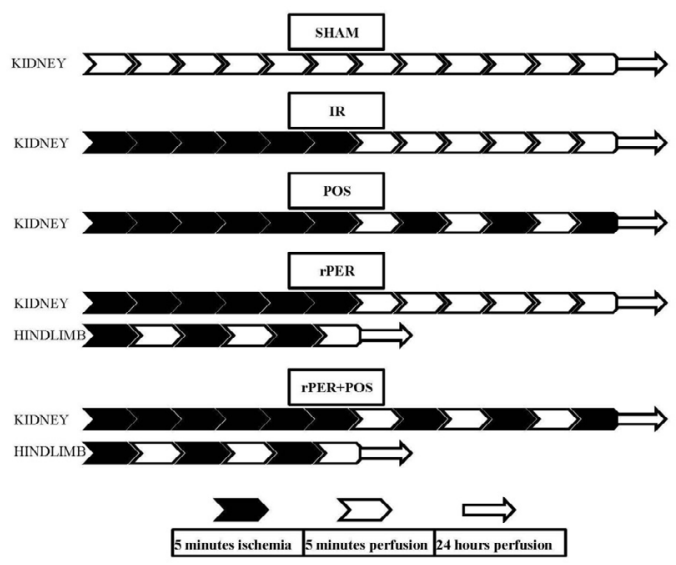

Figure 1 - The experimental protocol of each group.

\section{Surgical procedures}

Renal ischemia and remote and/or autologous per- and/or post-conditioning was performed in anesthesia (ketamine and xylazine $70 \mathrm{mg} / \mathrm{kg}$ and $10 \mathrm{mg} / \mathrm{kg}$, respectively, IP). Briefly, a right nephrectomy was performed and the left renal artery was exposed through a midline laparotomy. Renal ischemia was induced by applying a microsurgical clip on the renal artery. Hindlimb ischemia was achieved using an elastic rubber band tied around the thigh of the left leg, through a non-invasive method that was successfully used in previous studies $^{18,22}$.

$$
\text { Following the renal ischemia/ }
$$
conditioning protocols, rats were allowed to recover from anesthesia for 24 hours. Then rats were anesthetized and a $3 \mathrm{ml}$ blood sample was obtained via puncture of the abdominal vena cava and the left kidney was harvested for histological analysis. Subsequently, the animals were euthanized by lethal anesthetic doses. 


\section{Parameters evaluation}

Blood samples were immediately sent to laboratory analysis. Urea and creatinine levels were measured on Selectra-E auto analyzer. The left kidney was cut longitudinally in two halves, fixed in $10 \%$ formaldehyde, embedded in paraffin, and stained using hematoxyline/ eosine. Multiple sections were analyzed with regard to the glomerulus and tubules injury.

Total number of glomeruli per field as well as damaged glomeruli has been accounted for. Glomeruli were considered normal whenever capillary loops were open, with thin walls and whenever there was no content inside the Bowman's capsule. On the other hand, glomeruli were considered as damaged whenever they presented a contraction of glomerular tuft with approximation of structures, contents inside the Bowman's capsule or vacuolization of endothelial cells. The degree of glomerular lesion has been characterized as: 0- No damage; 1-light (up to $25 \%$ of damaged glomeruli); 2 -moderate (26\% to $50 \%$ of damaged glomeruli); 3-sharp (51\% to $75 \%$ of damaged glomeruli); severe (above $75 \%$ of damaged glomeruli $)^{25}$.

Tubules were considered damaged when there was either severe tubular lysis, loss of brush border, and sloughed debris in tubular lumen space. Tubular damage was graded at: 0: no damage; 1: 0-25\% damaged tubules; 2 : 25-50\% damaged tubules; 3: 50-75\% damaged tubules; 4 : $>75 \%$ damaged tubules ${ }^{25}$.

\section{Statistics}

Analysis of variance (ANOVA), followed by Tukey post-hoc tests correction, was performed to analyze urea and creatinine. Kruskal-Wallis, followed by Student-NewmanKeuls correction, was used to analyze the histological parameters. Statistical significance was assumed at $p<0.05$.

\section{Results}

During the procedure, no animal died or were performed resuscitation maneuvers. Table 1 shows the mean of urea and creatinine serum levels. From the urea serum level, IR showed the highest level when compared to all groups. PER and PER+POS obtained better results than IR and POS groups $(p<0.05)$, and not show difference in comparison to SHAM. POS obtained better results than IR $(p<0.05)$. PER and PER + POS groups obtained similar values $(p=0.49)$.

The IR group had the highest creatinine levels compared to all other groups $(p<0.001)$; POS group obtained worse results than the PER and PER + POS group $(p<0.01) ; P E R$ and PER + $P O S$ groups obtained similar values $(p=0.88)$.

Table 1 - Mean urea and creatinine serum level of each group.

\begin{tabular}{lll}
\hline Group & Urea & Creatinine \\
\hline Normality & $72.16 \pm 7.13$ & $0.30 \pm 0.30$ \\
Sham & $86.18 \pm 11.95$ & $1.14 \pm 0.34$ \\
Ischemia-Reperfusion & $216.30 \pm 7.17$ & $2.06 \pm 0.34$ \\
Postconditioning & $137.84 \pm 6.81$ & $1.38 \pm 0.21$ \\
Perconditioning & $83.74 \pm 14.58$ & $0.72 \pm 0.11$ \\
Per+Postconditioning & $100.24 \pm 12.40$ & $0.71 \pm 0.10$ \\
\hline p $<0.01$ Ischemia-Reperfusion vs. all groups \\
p $>0.05$ Perconditioning vs. Per+Postconditioning
\end{tabular}

Table 2 shows the results of the histopathological analysis (Figure 2). PER showed better glomerulus and tubules structure than the IR group $(p<0.01)$. POS showed only better glomerulus structure than the IR group $(p<0.01)$. PER+POS had no statistical difference when compared to the IR group in both glomerulus $(p=0.09)$ and tubules $(p=0.11)$ grading. 
Table 2 - Average histopathological scores.

\begin{tabular}{lll}
\hline Group & $\begin{array}{l}\text { Glomerular } \\
\text { lesion }\end{array}$ & $\begin{array}{l}\text { Tubular } \\
\text { damage }\end{array}$ \\
\hline Normality & $0.00 \pm 0.00$ & $0.00 \pm 0.00$ \\
Sham & $0.40 \pm 0.54$ & $0.40 \pm 0.89$ \\
Ischemia-Reperfusion & $3.80 \pm 0.44$ & $3.60 \pm 0.54$ \\
Postconditioning & $2.00 \pm 0.70$ & $2.80 \pm 0.83$ \\
Perconditioning & $1.80 \pm 0.44$ & $1.60 \pm 0.89$ \\
Per+Postconditioning & $2.40 \pm 0.89$ & $2.80 \pm 0.83$ \\
\hline
\end{tabular}

$p<0.01$ Ischemia-Reperfusion and Sham $v s$ all groups p $>0.05$ Perconditioning vs Per+Postconditioning

\section{- Discussion}

Perconditioning is the newest technique described to mitigate IR injury in many tissues ${ }^{15,18,20}$. Our study is the first evaluate of the potential protective effect of PER alone or combined with POS on kidney IR-induced injury, that only be test before in myocardium $^{15,20}$, brain ${ }^{17,26}$, and liver ${ }^{18}$.

Creatinine is a good measurement of kidney filtration being a parameter for glomerular function ${ }^{27}$. All tissue conditioning techniques were able to protect glomerular function. PER and PER+POS showed similar values. POS shows an urea serum level worse than PER. Since urea excretion occurs through glomerular filtration (40\%) and tubular secretion on Henle Loop $(60 \%)^{28}$, then we hypothesize that PER is giving protection to both glomerulus and tubules, but POS is only protecting glomerular function. The histological analysis shows results similar to those identified in the analysis of serum urea and creatinine and confirming the initial conclusions, similar data was find by Chen et al. ${ }^{29}$.

The vascular anatomy of kidney is unique $^{22,30}$ where glomerulus receive blood supply prior to medullar area (tubulus), being very different from the heart or liver vascular anatomy; myocites have also different cell biology. Our findings that PER might confer protection to the glomerulus and tubules and POS only to the glomerulus, support that tissue conditioning techniques might work in a tissue dependent manner.

We could clearly detect that there was no additional effects when both techniques were held together. The association had slightly worse outcome that could be of statistical significance in a larger series. We suggest that the complementary postconditioning cycles might lead to additional tissue damage, in opposition to the expected additional protection, or at least act in the same pathway, saturating $i t^{18}$. So, we can also conclude that PER is the best tissue conditioning for kidney ischemia and reperfusion syndrome.

Mechanisms underlying PER and POS protective effects are barely understood, they probably act in different pathway depending on the target organ. In brain ${ }^{27}$ and myocardium ${ }^{31}$, the combined of per and postconditioning showing a positive synergism; however, in liver ${ }^{18}$ there no additional effect, similar to the find of this study.

The finds of this study show that for kidney IR syndrome the perconditioning have a better protecting effect than postconditioning alone or combined. Based on fact that perconditioning is performed in a distant tissue, is a non-invasive procedure and do not increase the surgical time, thus does not require direct access to the artery and could be easily applied during the surgery or endovascular surgery ${ }^{15-19}$; this ischemic conditioning must be test in human IR syndromes ${ }^{20,31}$. However, new studies aim to understanding the mechanism are need ${ }^{32}$.

We tested three cycles of $5 \mathrm{~min}$ of reperfusion followed by $5 \mathrm{~min}$ ischemia, interval referred to previous literature ${ }^{18,22,32}$. Whether, the ischemic per and postconditioning plays its role in an 'on-off' style or a 'dose-dependent' one $22,32,33$ was not fully elucidated in this study. Five min may not afford maximal protective 
effect against renal IR injury if ischemic conditioning was a 'dose-dependent' one; and that can influence direct on the result of the combined effect, mainly if one conditioning is minimized. Thus, the exact number of optimal interval and cycles need to be investigated; and with this interval, new studies combining both techniques could be performed.

\section{Conclusions}

Perconditioning had promising results on ischemia and reperfusion induced kidney injury, enhanced kidney function and protected glomerulus and tubules. There was no additive protection when postconditioning and perconditioning were combined.

\section{References}

1. Yellon DM, Hausenloy DJ. Myocardial reperfusion injury. N Engl J Med. 2007;357:1121-35. doi: 10.1056/ NEJMra071667.

2. Holger KE, Tobias E. Ischemia and reperfusion-from mechanism to translation. Nat Med. 2001;17:1391-401. doi: $10.1038 / \mathrm{nm} .2507$.

3. Nolan CR, Anderson RJ. Hospital-acquired acute renal failure. J Am Soc Nephrol. 1998;9:710-8. PMID: 9555674.

4. Silva JR OC, Centurion S, Pacheco EG, Brisotti $\mathrm{JL}$, Oliveira AF, Dal Sasso K. Basics aspects of the ischemia reperfusion injury and of the ischemic preconditioning. Acta Cir Bras. 2002;17(3):96-100. doi: 10.1590/S010286502002000900020.

5. Brito MV, Yasojima EY, Percário S, Ribeiro Júnior RF, Cavalcante LC, Monteiro AM, Couteiro RP, Rodrigues IA, Santos HA. Effects of hypertonic saline solution associated to remote ischemic perconditioning in kidney ischemia/reperfusion injury in rats. Acta Cir Bras. 2017 Mar;32(3):211-8. doi: 10.1590/ S0102-865020170030000005.

6. Kuczmarski JM, Martens CR, LennonEdwards SL, Edwards DG. Cardiac function and tolerance to ischemia-reperfusion injury in chronic kidney disease. Nephrol
Dial Transplant. 2014;29(8):1514-24. doi: 10.1093/ndt/gft336.

7. Song $X$, Zhang N, XU H, Cao L, Zhang $\mathrm{H}$. Combined preconditioning and postconditioning provides synergistic protection against liver ischemic reperfusion injury. Int J Biol Sci. 2012;8(5):707-18. doi: 10.7150/ijbs.4231.

8. Zhang WX, Yin $W$, Zhang L, Wang LH, Bao L, Tuo HF. Preconditioning and postconditioning reduce hepatic ischemiareperfusion injury in rats. Hepatobiliary Pancreat Dis Int. 2009;8(6):586-90. PMID: 20007074.

9. Kerendi $F$, Kin $H$, Halkos ME, Jiang R, Zatta AJ, Zhao ZQ, Guyton RA, Vinten-Johansen J. Remote postconditioning. Brief renal ischemia and reperfusion applied before coronary artery reperfusion reduces myocardial infarct size via endogenous activation of adenosine receptors. Basic Res Cardiol. 2005;100:404-12. doi: 10.1007/ s00395-005-0539-2.

10.Er F, Nia AM, Dopp H, Hellmich M, Dahlem KM, Caglayan E, Kubacki T, Benzing T, Erdmann E, Burst V, Gassanov N. Ischemic preconditioning for prevention of contrast medium-induced nephropathy: randomized pilot RenPro Trial (Renal Protection Trial). Circulation. 2012;126(3):296-303. doi: 10.1161/CIRCULATIONAHA.112.096370.

11.Murry $C E$, Jennings $R B$, Reimer $K A$. Preconditioning with ischemia: a delay of lethal cell injury in ischemic myocardium. Circulation. 1986;74:1124-36. PMID: 3769170.

12.Chen $H$, Xing B, Liu X, Zhan B, Zhou J, Zhu H, Chen $Z$. Ischemic postconditioning inhibits apoptosis after renal ischemia/reperfusion injury in rat. Transpl Int. 2008;21:364. PMID: 18069925.

13.Zhao ZQ, Corvera JS, Halkos ME, Kerendi F, Wang NP, Guyton RA. Inhibition of myocardial injury by ischemic postconditioning during reperfusion: comparison with ischemic preconditioning. Am J Physiol Heart Circ Physiol. 2003;285:579-88. doi: 10.1152/ ajpheart.01064.2002.

14.Santos CHM, Gomes OM, Pontes JCDV, Miiji LNO, Bispo MAF. The ischemic preconditioning and postconditioning effect on the intestinal mucosa of rats undergoing mesenteric 
procedure. Acta Cir Bras. 2008;23(1):22-8. PMID: 18278389.

15.Schmidt MR, Smerup $M$, Konstantinov IE, Shimizu M, Li J, Cheung. Intermittent peripheral tissue during coronary ischemia reduces myocardial infarction through a KATP-dependent mechanism: first demonstration of remote ischemic perconditioning. Am J Physiol Heart Circ Physiol. 2007;292:1883-90. doi: 10.1152/ ajpheart.00617.2006.

16. Hahn CD, Manlhiot C, Schmidt MR, Nielsen $\mathrm{TT}$, Redington AN. Remote ischemic preconditioning: a novel therapy for acute stroke. Stroke. 2011;42:2960-2. doi: 10.1161/STROKEAHA.111.622340.

17. Hoda MN, Siddiqui S, Herberg S, PeriyasamyThandavanS, Bhatia K, HafezSS, Johnson MH, Hill WD, Ergul A, Fagan SC, Hess DC. Remote ischemic perconditioning is effective alone and in combination with intravenous tissuetype plasminogen activator in murine model of embolic stroke. Stroke. 2012;43(10):27949. doi: 10.1161/STROKEAHA.112.660373.

18.Costa FL, Yamaki VN, Gonçalves TB, Coelho JV, Percário S, Brito MV. Combined remote ischemic perconditioning and local postconditioning on liver ischemiareperfusion injury. J Surg Res. 2014;192:98102. doi: 10.1016/j.jss.2014.05.046.

19.Wei M, Xin P, Li S, Tao J, Li Y, Li J, Liu M, Li J, Zhu W, Redington AN. Repeated remote ischemic postconditioning protects against adverse left ventricular remodeling and improves survival in a rat model of myocardial infarction. Circ Res. 2013;108(10):1220-5. doi: $10.1161 /$ CIRCRESAHA.110.236190.

20. Hougaard KD, Hjort N, Zeidler D, Sørensen L, Nørgaard A, Hansen TM, von WeitzelMudersbach $P$, Simonsen CZ, Damgaard D, Gottrup H, Svendsen K, Rasmussen PV, Ribe LR, Mikkelsen IK, Nagenthiraja K, Cho $\mathrm{TH}$, Redington AN, Bøtker HE, Ostergaard L, Mouridsen K, Andersen G. Remote ischemic perconditioning as an adjunct therapy to thrombolysis in patients with acute ischemic stroke: a randomized trial. Stroke. 2014;45:159-67. doi: 10.1161/ STROKEAHA.113.001346.

21.Xin P, Zhu W, Li J, Ma S, Wang L, Liu $M$, Redington AN. Combined local ischemic postconditioning and remote ischemic preconditioning recapitulate cardioprotective effects of local ischemic preconditioning. Am J Physiol Heart Circ Physiol. 2010;298:1819-31. doi: 10.1152/ ajpheart.01102.2009.

22. Yamaki VN, Gonçalves TB, Coelho JV, Pontes RV, Costa FL, Brito MV. Protective effect of remote ischemic per-conditioning in the ischemia and reperfusion-induce renal injury in rats. Rev Col Bras Cir. 2012;39(6):529-33. doi: 10.1590/S0100-69912012000600014.

23.Tamareille S, Mateus V, Ghaboura N, Jeanneteau J, Croué A, Henrion D. RISK and SAFE signaling pathway interactions in remote limb ischemic perconditioning in combination with local ischemic postconditioning. Basic Res Cardiol. 2011;37:1-11. doi: 10.1007/s00395-0110210-z.

24.Donato $M$, Buchholz $B$, Rodríguez $M$, Pérez V, Inserte J, García-Dorado D, Gelpi RJ. Role of the parasympathetic nervous system in cardioprotection by remote hindlimb ischaemic preconditioning. Exp Physiol. 2013;98(2):425-34. doi: 10.1113/ expphysiol.2012.066217.

25. Meyer F, Lizana JN, Dziedricki LF, BleggiTorres LF. Histologic alterations of rat kidneys perfused with a Euro-Collins diltiazem solution. Acta Cir Bras. 2010;25(6):496-500. doi: 10.1590/S0102-86502010000600007.

26.Ren C, Wang P, Wang B, Li N, Li W, Zhang C, Jin K, Ji X. Limb remote ischemic perconditioning in combination with postconditioning reduces brain damage and promotes neuroglobin expression in the rat brain after ischemic stroke. Restor Neurol Neurosci. 2015;33(3):369-79. doi: 10.3233/ RNN-140413.

27.Passos MT, Nishida SK, Câmara NO, Shimizu $\mathrm{MH}$, Mastroianni-Kirsztajn G. Lohexol clearance for determination of glomerular filtration rate in rats induced to acute renal failure. Plos One. 2015;10(4):e0123753. doi: 10.1371/journal.pone.0123753.

28.Pagana KD, Pagana TJ. Urea nitrogen, Blood. In: Pagana KD, Pagana TJ, editors. Mosby`s diagnostic \& laboratory test reference. 11ed. St. Louis: Mosby; 2011. p.131-3.

29. ChenH, Wang L, Xing BZ, Liu XH, ChenZY, Weng $X D$, Qiu T, Liu L. Ischemic postconditioning attenuates inflammation in rats following renal ischemia and reperfusion injury. Exp Ther Med. 2015 Aug;10(2):513-8. doi: 
10.3892/etm.2015.2514.

30.Yamaki IN, Pontes RV, Costa FL, Yamaki VN, Teixeira RK, Yasojima EY, Brito MV. Kidney ischemia and reperfunsion syndrome: effect of lidocaine and local postconditioning. Rev Col Bras Cir. 2016 Sep;43(5):348-53. doi: 10.1590/0100-69912016005012.

31.Eitel I, Stiermaier T, Rommel KP, Fuernau G, Sandri M, Mangner N, Linke A, Erbs S, Lurz P, Boudriot E, Mende M4, Desch S, Schuler G, Thiele H. Cardioprotection by combined intrahospital remote ischaemic perconditioning and postconditioning in ST-elevation myocardial infarction: the randomized LIPSIA CONDITIONING trial. Eur Heart J. 2015 Nov 21;36(44):3049-57. doi: 10.1093/eurheartj/ehv463.

32. Costa FL, Teixeira RK, Yamaki VN, Valente AL, Silva AM, Brito MV, Percário S. Remote ischemic conditioning temporarily improves antioxidant defense. J Surg Res. 2016 Jan;200(1):105-9. doi: 10.1016/j. jss.2015.07.031.

33. Chen H, Xing B, Liu X, Zhan B, Zhou J, Zhu H, Chen $Z$. Ischemic postconditioning inhibits apoptosis after renal ischemia/reperfusion injury in rat. Transpl Int. 2008 Apr;21(4):36471. doi: 10.1111/j.1432-2277.2007.00606.x.

\section{Correspondence:}

Renan Kleber Costa Teixeira

Rua Mundurucus, 2256/1401

66035-360 Belém - PA Brasil

Tel.: (55 91)98145-1108

renankleberc@hotmail.com

Received: Apr 13, 2017

Review: June 14, 2017

Accepted: July 17, 2017
Conflict of interest: none

Financial source: none
${ }^{1}$ Research performed at Laboratório de Cirurgia Experimental, Universidade do Estado do Pará (UEPA), Belem-PA, Brazil. 